Chapter 2

\title{
Pleural Photodynamic Therapy and Surgery in Thoracic Cancer Patients with Pleural Spread
}

\author{
Ke-Cheng Chen and Jang-Ming Lee \\ Additional information is available at the end of the chapter
}

http://dx.doi.org/10.5772/intechopen.68722

\begin{abstract}
Pleural spread from non-small cell lung cancer is a difficult situation. The average survival in the situation is about 6-9 months. We investigate the current management of this challenging condition. Although, there is no much evidence found in the literature, we do see the pleural photodynamic therapy giving some promising light in the dark night. However, the patients still require complete neoadjuvant and adjuvant therapies, as well as radical tumor resection. Pleural PDT is one of the multi-modality treatments, which combined can achieve satisfactory oncological results. The long-term survival can be achieved in more than half the patients. However, the side effects of pleural PDT include skin hypersensitivity, trachea and esophageal perforation, and ARDS, which we should keep in mind.
\end{abstract}

Keywords: PDT, lung cancer, thymic cancer, pleural spread

\section{Introduction}

Photodynamic therapy (PDT) is one of the established treatment modality for non-small cell lung cancer (NSCLC). Early-stage lung cancer and superficial endobronchial lesions less than $1 \mathrm{~cm}$ in thickness can be effectively treated with external light sources. Thicker lesions and peripheral lesions may be amenable to interstitial PDT, where the light is delivered intratumorally. The primary adverse event, phototoxicity, is expected to be minimized with the introduction of new photosensitizers that have shown promising results in phase I and II clinical studies. Moreover, the addition of PDT to standard-of-care surgery and chemotherapy (or target therapy) can improve survival and outcomes in patients with pleural dissemination. Therefore, intra-operative PDT has shown promise in the treatment of non-small cell lung cancer with pleural spread. 
The presence of pleural spread in non-small cell lung cancer (NSCLC) without distant metastases was classified as stage III b in the previous International System for Staging Lung Cancer [1]. The seventh edition of the TNM classification of lung cancer was published in 2009, and the changes to the sixth edition of this document were according to proposals from the International Association for the Study of Lung Cancer (IASLC). The IASLC lung cancer staging project committee suggested that pleural disseminations (pleural nodules or malignant pleural effusions) be reclassified to M1a from T4. In patients with pleural carcinomatosis, the reported median survival time ranged from 6 to 9 months [2-8]. Currently, the management options for pleural spread include chemotherapy, surgery with pleurectomy, and photodynamic therapy. Thymomas are neoplasms arising from epithelial thymic cells. They rarely metastasize to distant sites; on the contrary, they more often show pleural implantation at diagnosis or during follow-up. Thymoma with pleural spread is also a difficult clinical situation to manage, and the treatment is controversial [9-11].

Photodynamic therapy (PDT) is an anticancer treatment combining photosensitizer, oxygen, and visible light. PDT anticancer effect occurs when the photosensitizer captures light energy and transfers that energy to oxygen. The excited oxygen species are responsible for the effect and can directly kill tumor cells, damage the tumor blood supply, or both [10]. Porfimer sodium (Photofrin; Axcan Pharma Inc, Birmingham, AL, USA), a first-generation photosensitizer that is a mixture of porphyrin monomers and oligomers, is used most often. They are activated by $630 \mathrm{~nm}$ red light. Successful treatment of malignant mesothelioma by photodynamic therapy has been reported as a new approach for pleural malignancy dissemination [12]. Moreover, as with malignant pleural mesothelioma, PDT may be utilized as part of a multi-modality management strategy for non-small cell lung cancer with pleural spread. A phase II trial enrolling 22 patients with pleural spread and clinical T4 NSCLC was conducted in which patients underwent surgery with complete or partial tumor debulking, followed by hemithoracic pleural PDT or PDT alone. The median overall survival was 21.7 months, compared with 6-9 months for similar patients based on historical controls [13].

\section{Operative procedures}

Thoracotomy was performed for either multiple wedge resection or lobectomy. The particular pulmonary resection was chosen by the same criteria as those used for performing resections with curative intent in patients with early lung cancer. Anatomic resections (pneumonectomy, lobectomy, or segmentectomy) were performed only in patients in whom it was possible to resect all gross disease. After finishing lung resection, total parietal pleurectomy was performed subsequently. The parietal pleura were stripped from the bony hemithorax as radical parietal pleurectomy. The mediastinum was debulked of all gross tumor. For thymoma patients, the radical thymothymectomy was performed concurrent with radical pleural pleurectomy. The goal was to have no visible or palpable tumor left in the affected hemithorax at the end of the operation [14].

We sewed flat photodiodes into seven regions of the pleural space, which include the apex, anterior chest wall, posterior chest wall, posterior costophrenic sulcus, anterior costophrenic sulcus, posterior mediastinum, and pericardium. Moreover, we handle it with a dosimetry system. The dosimetry system provided both the real-time luminescence and the cumulative 
light dose for the seven regions. The cavity was filled with diluted intralipid solution $(0.01 \%)$ to act as scattering agent, giving a more homogeneous light delivery. The light was delivered with an optical fiber sheathed within a modified endotracheal tube. This delivery system was moved around the chest cavity until a measured dose of $40 \mathrm{~J} / \mathrm{cm}^{2}$ of $630 \mathrm{~nm}$ light was recorded at all seven regions (Figures 1 and 2). During the light delivery of PDT, the chest retractors were removed to avoid shielding. We use $20 \mathrm{~L}$ of intralipid solution to maintain a clear intrathoracic area and to minimize light absorption affected by hemoglobin. Sterile photodiodes were removed from the pleural cavity after completion of light administration. The overall time of the light delivery portion of PDT was about $1 \mathrm{~h}$. Then, the operation is completed after wound closure and setting two $28 \mathrm{fr}$ chest tubes.

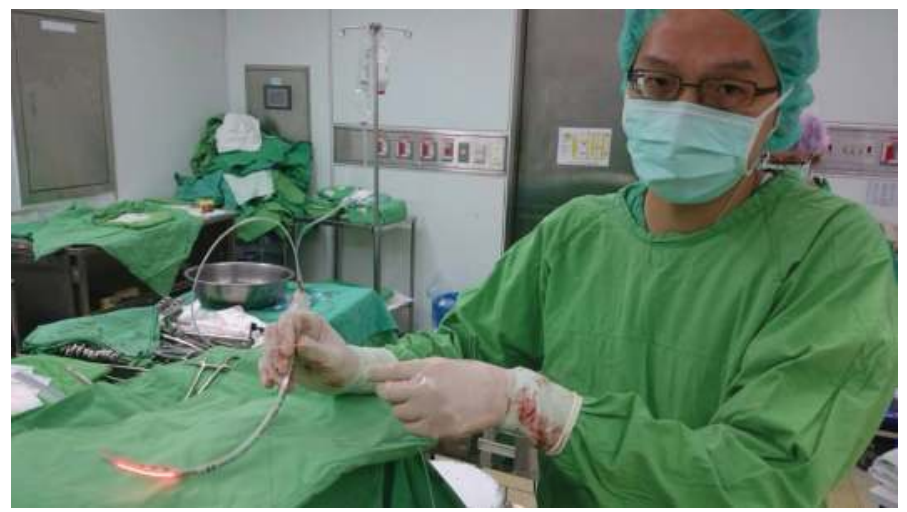

Figure 1. The operator and the $630 \mathrm{~nm}$ light source. The operator is hoding the laser light source which can activate the photosensitizer in tumor cells. The laser probe is within the endotracheal tube which enhance the convenience of irradiation in the pleural space.

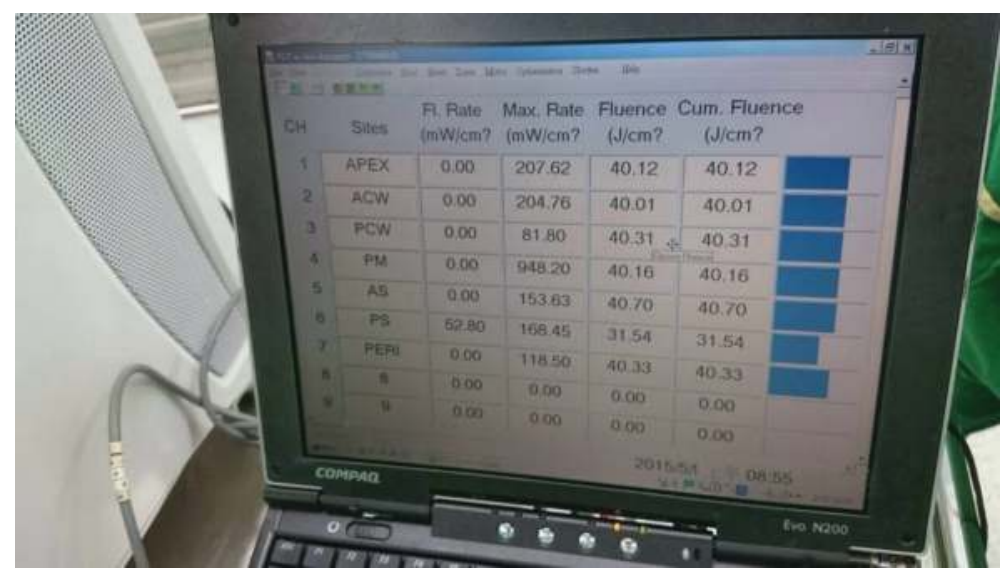

Figure 2. The dosimetry system. The dosimetry system provided both the real-time luminescence and the cumulative light dose for the seven regions. 


\section{Comments}

The pleural spread of thoracic malignancy is difficult to manage. The standard care of the condition is non-operative worldwide. Currently, surgery is not considered part of the treatment for these patients. It is usually treated with palliative chemotherapy but is barely cured. Surgery alone has been ineffective in treating cancer with pleural dissemination because residual microscopic disease remains in the chest cavity, even after what appears to be a "complete" resection [2-8]. Photodynamic therapy offers several potential advantages for treating disseminating surface cancer. First, preclinical studies demonstrate a greater retention of photosensitizers in tumor compared with normal tissues [15]. Second, the PDT penetrates several millimeters into tissue. It results in surface cell killing, while sparing the underlying tissues. Therefore, PDT is suitable for the treatment of cancers that have spread to organ surfaces, including pleura or peritoneum $[16,17]$. Moreover, PDT is a localized therapy that can be performed intraoperatively. Intraoperative pleural PDT was used as one of the multimodal approaches for treating patients with pleural carcinomatosis. The hypothesis was that, upon complete resection of all gross disease, immediate intraoperative PDT might be effective in treating the residual microscopic disease. For lung cancer, the phase II trial at the University of Pennsylvania proved our point of view. It showed $73.3 \%$ of 6 -month localized disease control for the cohort and a median overall survival of 21.7 months, compared with 6-9 months survival for similar patients treated with the non-operative standard of care and based upon historical controls [13]. Compared with surgery without PDT for patients of lung cancer with pleural spread in literature, the outcome of patients receiving surgery plus PDT is better $[18,19]$. The best median overall survival of the lung cancer patients was 39.0 months, conducted by our group (Figure 3) [20]. The comparison of these results is listed in Table 1 . Therefore, we found that the result was good, and the morbidity was acceptable. However, the PDT is only one of the multi-modality treatments, so the adjuvant or neoadjuvant therapy is important, too. Our group conducted another experiment about the significance of the Epidermal Growth Factor Receptor (EGFR) profile to the PDT-sensitive cancer cells [21]. Because tyrosine kinase inhibitor (TKI) was popularly used in the advanced lung cancer, we investigated the roles of this two common therapies combined. Although photodynamic therapy (PDT) has been demonstrated to be an effective minimally invasive treatment modality for thoracic cancer, the molecular action in thoracic cancer during PDT is hardly known. EGFR has been known to downregulate in various cancer cells during PDT. In the study, we investigated the effects of Photofrin-mediated PDT on cell death and expression of EGFR in CE48T/ VGH (CE48T) squamous carcinoma cells. We found that the photosensitizer Photofrin in the absence of light exposure can downregulate the expression of EGFR at both transcription and translation levels. Higher concentrations of Photofrin result in cytotoxicity, whereas lower doses of Photofrin inhibit EGFR expression under dark control without inducing significant cell death. This Photofrin-associated inhibition of EGFR was repeated in lung cancer, cervical cancer, and glioblastoma cells. Another squamous cell carcinoma cell line CE81T/VGH (CE81T) was found to be resistant to Photofrin-induced inhibition of EGFR as well as to Photofrin-mediated dark toxicity compared with CE48T. The resistance to the cytotoxicity in CE81T cells became insignificant when the Photofrin-treated cells were further irradiated by red light (Photofrin-PDT). We suggest Photofrin modulates the expression of EGFR in cancer cells. However, efficient cell death still requires the combination of Photofrin and light irradiation in squamous cell carcinoma cells. 


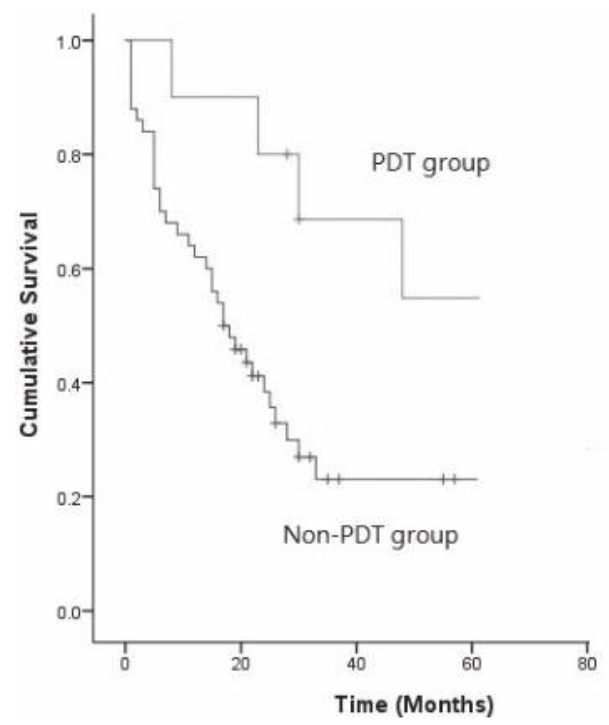

Figure 3. Kaplan-Meier survival analysis of the patients undergoing PDT vs. non-PDT for pleural spread $(\mathrm{P}=0.047)$.

\begin{tabular}{llllll}
\hline Studies & Year & Country & Mean age \pm SD (years) & No. of patients & 5-year survival rate (\%) \\
\hline Wang et al. & 2011 & Taiwan & $62.3 \pm 11.2$ & 90 & 21.7 \\
Mordant et al. & 2011 & France & $59.0 \pm 8.8$ & 32 & 16.0 \\
Chen et al. & 2013 & Taiwan & $51.9 \pm 11.9$ & 10 & 56.3 \\
\hline
\end{tabular}

Table 1. Comparison with other series for lung cancer patients.

\section{Conclusion}

Photodynamic therapy is a promising treatment modality for thoracic malignancy. With proper patient selection, intrapleural photodynamic therapy for pleural spread in patients with lung cancer or thymoma is feasible and may provide a survival benefit.

\section{Author details}

Ke-Cheng Chen and Jang-Ming Lee*

*Address all correspondence to: jangminglee@gmail.com

Department of Surgery, National Taiwan University Hospital, Taipei, Taiwan 


\section{References}

[1] Mountain CF. Revisions in the international system for staging lung cancer. Chest. 1997;111(6):1710-1717

[2] Werner-Wasik M, Scott C, Cox JD, Sause WT, Byhardt RW, et al. Recursive partitioning analysis of 1999 Radiation Therapy Oncology Group (RTOG) patients with locally advanced non-small-cell lung cancer (LANSCLC): Identification of five groups with different survival. International Journal of Radiation Oncology Biology Physics. 2000;48(5): 1475-1482

[3] Martini N, Bains MS, Beattie EJ Jr. Indications for pleurectomy in malignant effusion. Cancer. 1975;35(3):734-738

[4] Reyes L, Parvez Z, Regal AM, Takita H. Neoadjuvant chemotherapy and operations in the treatment of lung cancer with pleural effusion. The Journal of Thoracic and Cardiovascular Surgery. 1991;101(5):946-947

[5] Mott FE, Sharma N, Ashley P. Malignant pleural effusion in non-small cell lung cancer-time for a stage revision? Chest. 2001;119(1):317-318

[6] Rami-Porta R, Ball D, Crowley J, Giroux DJ, Jett J, et al. The IASLC Lung Cancer Staging Project: Proposals for the revision of the T descriptors in the forthcoming (seventh) edition of the TNM classification for lung cancer. Journal of Thoracic Oncology. 2007;2(7):593-602

[7] Postmus PE, Brambilla E, Chansky K, Crowley J, Goldstraw P, et al. The IASLC Lung Cancer Staging Project: Proposals for revision of the M descriptors in the forthcoming (seventh) edition of the TNM classification of lung cancer. Journal of Thoracic Oncology. 2007;2(8):686-693

[8] Goldstraw P, Crowley J, Chansky K, Giroux DJ, Groome PA, et al. The IASLC Lung Cancer Staging Project: Proposals for the revision of the TNM stage groupings in the forthcoming (seventh) edition of the TNM Classification of malignant tumours. Journal of Thoracic Oncology. 2007;2(8):706-714

[9] Lucchi M, Davini F, Ricciardi R, Duranti L, Boldrini L, et al. Management of pleural recurrence after curative resection of thymoma. The Journal of Thoracic and Cardiovascular Surgery. 2009;137(5):1185-1189

[10] Yu L, Jing Y, Ma S, Li F, Zhang YF. Cytoreductive surgery combined with hyperthermic intrapleural chemotherapy to treat thymoma or thymic carcinoma with pleural dissemination. OncoTargets and Therapy. 2013;6:517-521

[11] Ishikawa Y, Matsuguma H, Nakahara R, Suzuki H, Ui A, et al. Multimodality therapy for patients with invasive thymoma disseminated into the pleural cavity: The potential role of extrapleural pneumonectomy. The Annals of Thoracic Surgery. 2009;88(3):952-957 
[12] Friedberg JS, Culligan MJ, Mick R, Stevenson J, Hahn SM, et al. Radical pleurectomy and intraoperative photodynamic therapy for malignant pleural mesothelioma. The Annals of Thoracic Surgery. 2012;93(5):1658-1665

[13] Friedberg JS, Mick R, Stevenson JP, Zhu T, Busch TM, et al. Phase II trial of pleural photodynamic therapy and surgery for patients with non-small-cell lung cancer with pleural spread. Journal of Clinical Oncology. 2004;22(11):2192-2201

[14] Liu TJ, Lin MW, Hsieh MS, Kao MW, Chen KC, et al. Video-assisted thoracoscopic surgical thymectomy to treat early thymoma: A comparison with the conventional transsternal approach. Annals of Surgical Oncology. 2014;21(1):322-328

[15] Gomer CJ, Dougherty TJ. Determination of [3H]- and [14C]hematoporphyrin derivative distribution in malignant and normal tissue. Cancer Research. 1979;39(1):146-151

[16] Dougherty TJ, Gomer CJ, Henderson BW, Jori G, Kessel D, et al. Photodynamic therapy. Journal of the National Cancer Institute. 1998;90(12):889-905

[17] Hahn S, Glatstein E. The emergence of photodynamic therapy as a major modality in cancer treatment. Reviews in Contemporary Pharmacotherapy. 1999;10:69-74

[18] Wang BY, Wu YC, Hung JJ, Hsu PK, Hsieh CC, et al. Prognosis of non-small-cell lung cancer with unexpected pleural spread at thoracotomy. Journal of Surgical Research. 2011;169(1):e1-e5

[19] Mordant P, Arame A, Foucault C, Dujon A, Le Pimpec Barthes F, et al. Surgery for metastatic pleural extension of non-small-cell lung cancer. European Journal of CardioThoracic Surgery. 2011;40(6):1444-1449

[20] Chen KC, Hsieh YS, Tseng YF, Shieh MJ, Chen JS, Lai HS, Lee JM. Pleural photodynamic therapy and surgery in lung cancer. Plos One. 2015;10(7):e0133230. DOI: 10.1371/journal. pone.0133230. eCollection 2015

[21] Yang P-W, Hung M-C, Hsieh C-Y, Tung E-C, Wang Y-H, Tsai J-C, Lee J-W. The effects of Photofrin-mediated photodynamic therapy on the modulation of EGFR in esophageal squamous cell carcinoma cells. Lasers in Medical Science. 2013;28(2):605-614. DOI: 10.1007/s10103-012-1119-y. Epub 2012 May 15 
